\title{
Andrographolide inhibits multiple myeloma cells by inhibiting the TLR4/NF-кB signaling pathway
}

\author{
$\mathrm{HUI} \mathrm{GAO}^{1}$ and JIANRONG WANG ${ }^{2}$ \\ Departments of ${ }^{1}$ Hematology and ${ }^{2}$ Obstetrics, Dongying People's Hospital of Shandong, \\ Dongying, Shandong 257091, P.R. China
}

Received January 11, 2015; Accepted October 21, 2015

DOI: $10.3892 / \mathrm{mmr} .2015 .4703$

\begin{abstract}
Andrographolide is an active component from the extract of Andrographis paniculata [(Burm.f) Nees], a medicinal plant from the Acanthaceae family. Pharmacological studies have revealed that andrographolide possesses anti-bacterial, anti-inflammatory, anti-viral, immune regulatory and hepatoprotective properties, and is efficacious in the treatment of cardiovascular diseases, while exhibiting low toxicity and low cost. The present study aimed to determine the inhibitory effects of andrographolide on the growth of multiple myeloma (MM) cells and its possible impact on the Toll-like receptor (TLR)4/nuclear factor (NF)- $\mathrm{kB}$ signaling pathway. Cell proliferation was detected using an MTT assay, cellular apoptosis was measured using flow cytometry, and caspase-9/3 activation were assessed using colorimetric assay kits. Furthermore, TLR4 and NF- $\mathrm{kB}$ protein expression was determined by western blot analysis. The results revealed that andrographolide reduced the proliferation, while increasing cellular apoptosis and caspase-9/3 activation of MM cells, in addition to downregulating the expression of TLR4 and NF- $\kappa B$ protein. Of note, TLR4- or NF- $\mathrm{KB}$-targeting small-interfering (si)RNA enhanced the andrographolide-induced inhibition of cell proliferation and induction of apoptosis of MM cells. The results of the present study therefore suggested that andrographolide inhibited multiple myeloma cells via the TLR4/NF- $\mathrm{\kappa B}$ signaling pathway.
\end{abstract}

\section{Introduction}

Multiple myeloma (MM) is a malignant blood cancer type with the characteristic of plasma-cell clonal proliferation, which accounts for $\sim 10 \%$ of all hematological malignancies and has a yearly increasing incidence rate (1). In recent years,

Correspondence to: Mr. Hui Gao, Department of Hematology, Dongying People's Hospital of Shandong, 317 Dongcheng South First Road, Dongying, Shandong 257091, P.R. China

E-mail: huigaoqj@126.com

Key words: andrographolide, multiple myeloma, Toll-like receptor 4, nuclear factor- $\kappa \mathrm{B}$ with the application of novel chemotherapeutic drugs and improvements in treatment methods, as well as progress in the development and optimization of supportive treatments, $50-70 \%$ of patients receive effective chemotherapy; however, multiple cycles of chemotherapeutic treatments cause drug resistance, leading to refractory MM (2).

Studies on Drosophila (D.) melanogaster have led to the discovery of the Toll gene, which mainly determines the developmental direction of the front and lateral body axes in D. melanogaster as well as the non-specific immune response. Toll genes encode Toll-like receptors (TLR); the first TLR identified on the human cell surface displaying homology with D. melanogaster TLRs was TLR4 (3). Studies have shown that signaling pathways induced by TLRs, including TLR4 and TLR9, are important in tumor formation, and that the upregulation of TLRs may be closely associated with the development of cancer types, including gastric and colon cancer (4).

Nuclear factor (NF)- $\mathrm{\kappa B}$ is a key nuclear transcription factor which, under normal conditions, exists in the inactive forms of homologous or heterodimers in the cytoplasm of almost all types of cells, and which is associated with multiple cellular activities, including the activation of immune cells, development of T- and B-lymphocytes, stress response and cell apoptosis (5). Recent studies have shown that NF-kB is closely associated with the occurrence of hematopoietic malignancies, including leukemia, lymphoma and MM (6).

Andrographolide (Fig. 1) is a diterpene lactone compound extracted from Andrographis paniculata [(Burm.f) Nees], a medicinal plant from the Acanthaceae family, and is one of the major active components of the traditional Chinese medicine Andrographis with a content of up to $1.8 \%$ in the leaves (7). In China, Andrographis is being mass-produced as a raw material for the isolation of andrographolide used as an anti-inflammatory drug in formulations including Kalii Dehydrographolidi Succinas and Andrographis injection (8). Pharmacological studies have shown that andrographolide has anti-inflammatory, anti-bacterial, anti-viral, anti-tumor, immunoregulatory and hepato- and gallbladder-protective effects, as well as beneficial effects on cardiovascular diseases, with characteristics of low toxicity and low cost (9-12). However, to date, the potential of andrographolide to be used in the treatment of human MM has not been studied. The present study provided experimental evidence for the anti-cancer efficacy of andrographolide on MM cells; in 
addition, the mechanism of action and potential regulatory molecules involved, including TLR4 and NF- $\mathrm{KB}$, were assessed.

\section{Materials and methods}

Reagents. Dulbecco's modified Eagle' medium (DMEM) was obtained from Gibco (Thermo Fisher Scientific, Waltham, MA, USA). Fetal bovine serum (FBS) was purchased from Thermo Fisher Scientific. 3-(4,5-dimethylthylthiazol-2-yl)-2,5-diphenyltetrazolium bromide (MTT) was provided by Invitrogen (Thermo Fisher Scientific). Annexin V/propidium iodide (PI) was purchased from eBioscience (San Diego, CA, USA). Caspase-9/3 activation ELISA colorimetric assay kits were obtained from Santa Cruz Biotechnology, Inc. (Dallas, TX, USA). The bicinchoninic acid (BCA) Protein Assay kit was purchased from Beyotime Institute of Biotechnology (Jiangsu, China).

Cells and cell culture. The OPM1 human myeloma cell line was purchased from Shanghai Cell Bank (Shanghai, China) and cultured in complete DMEM with $10 \%$ heat-inactivated FBS, $100 \mathrm{U} / \mathrm{ml}$ penicillin and streptomycin $(100 \mu \mathrm{g} / \mathrm{ml}$; Sigma-Aldrich, St. Louis, MO, USA) at $37^{\circ} \mathrm{C}$ in a humidified atmosphere containing $5 \% \mathrm{CO}_{2}$.

Cell viability assay. OPM1 cells were seeded into 96 -well plates at $1 \times 10^{4} /$ well and allowed to attach overnight, following which they were treated with $1.0,5.0$ or $10.0 \mu \mathrm{M}$ andrographolide (Sigma-Aldrich; purity, $>98 \%$ ) for 24,48 or $72 \mathrm{~h}$ according to the procedure of a previous study (13). Subsequently, $20 \mu \mathrm{l}$ MTT $(5 \mathrm{mg} / \mathrm{ml})$ was added to each well and plates were cultured for an additional $4 \mathrm{~h}$, followed by aspiration of the media, addition of $150 \mu 1$ dimethylsulfoxide (Invitrogen; Thermo Fisher Scientific) to each well and agitation for $20 \mathrm{~min}$. The absorbance values were determined at $550 \mathrm{~nm}$ using an automatic microplate reader (Wallac Victor 1420; PerkinElmer, Inc., Waltham, MA, USA).

Flow cytometric analysis. OPM1 cells were inoculated into six-well plates at $2 \times 10^{6} /$ well and treated with $1.0,5.0$ and $10.0 \mu \mathrm{M}$ andrographolide for $24 \mathrm{~h}$. Each well was washed twice with phosphate-buffered saline (PBS) and following trypsinization (Beyotime Institute of Biotechnology), cells were suspended in $1 \mathrm{ml}$ binding buffer. Annexin V (5 $\mu \mathrm{l})$ was added and cells were incubated for $15 \mathrm{~min}$ in the dark. Subsequently, $5 \mu 1$ PI was added and cells were incubated for $30 \mathrm{~min}$ in the dark on ice. The apoptotic rate of OPM1 cells was then assessed by flow cytometry (FACSCalibur; BD Biosciences) with $1 \times 10^{6}$ events recorded.

Caspase-9/3 activation. OPM1 cells were seeded into 96-well plates at $1 \times 10^{4} /$ well and treated with $1.0,5.0$ or $10.0 \mu \mathrm{M}$ andrographolide for $24 \mathrm{~h}$. Caspase-9/3 activation in OPM1 cells was determined using ELISA colorimetric assay kits. Caspase-9 inhibitor LEHD-pNA and caspase-3 inhibitor Ac-DEVD-pNA were added to each well, and caspase-9/3 activation-associated fluorescence was detected at the wavelength of $405 \mathrm{~nm}$ using an automatic microplate reader (Wallac Victor 1420).

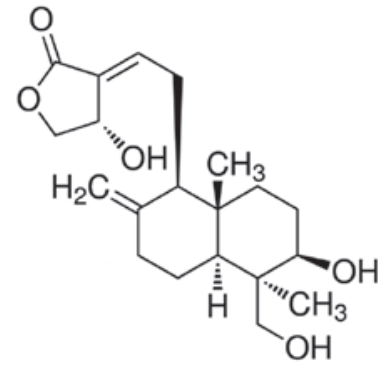

Figure 1. Chemical structure of andrographolide.

Western blot analysis. OPM1 cells were inoculated into six-well plates at $2 \times 10^{6} /$ well and treated with $1.0,5.0$ or $10.0 \mu \mathrm{M}$ andrographolide for $24 \mathrm{~h}$. Each well was washed twice with PBS and incubated with ice-cold lysis buffer (Beyotime Institute of Biotechnology) for $30 \mathrm{~min}$ on ice. The protein contents were determined using the BCA Protein Assay kit. Following loading of $10 \mu \mathrm{g}$ protein per lane, total protein was fractionated by $10 \%$ SDS-PAGE and transfer onto a polyvinylidene difluoride membrane at $4^{\circ} \mathrm{C}$ over $2 \mathrm{~h}$. Membranes were blocked with $5 \%$ non-fat dry milk in Tris-buffered saline (TBS) containing $0.05 \%$ Tween-20 prior to incubation with anti-TLR4 (cat. no. sc-293072; 1:1,000; Santa Cruz Biotechnology, Inc.); anti-NF-кB (cat. no. sc-56735; 1:1,000; Santa Cruz Biotechnology, Inc.) and $\beta$-actin (cat. no. AC106; 1:1,000; Beyotime Institute of Biotechnology, Inc.) overnight at $4^{\circ} \mathrm{C}$ with agitation. After extensive washing, membranes were incubated with secondary antibody (1:3,000; Tiangen, Beijing, China) for enhanced chemiluminescence (ECL) detection using Pierce ECL Western Blotting substrate (cat. no. 32109; Thermo Fisher Scientific).

Transfection of TLR4 small interfering (si)RNA and

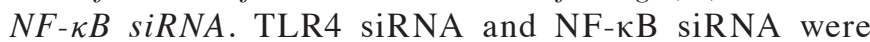
chemically synthesized by BeastBio Co., Ltd. (Shanghai, China). The siRNA sequences were as follows: TLR4 5'-GATCCCGACTTACAGTTTCTACGTTT CAAGAGAACGTAGAAACTGTAAGTCGTTA-3' and 5'-AG CTTAACGACTTACAGTTTCTACGTTCTCTTGAAACGT AGAAACTGTAAGTCGG-3'; and NF-кB: 5'-CCCCTTCCA AGTTCCTATA-3' and 5'-GGACATATGAGACCTTCAA-3'. OPM1 cells were seeded into six-well plates at $2 \times 10^{6} /$ well. 100 pmol TLR4 siRNA or NF-kB siRNA were transfected into OPM1 cells with Lipofectamine 2000 (Invitrogen) according to the manufacturer's instructions.

Statistical analysis. SPSS 17.0 software (SPSS, Inc., Chicago, IL, USA) was used for all statistical analyses. All quantitative values were obtained from experiments performed at least three times. Values are expressed as the mean \pm standard deviation. Statistical significance was analyzed using Student's t-test. $\mathrm{P}<0.05$ was considered to indicate a statistically significant difference.

\section{Results}

Andrographolide inhibits the proliferation of MM cells. To investigate whether andrographolide inhibited the proliferation 
of MM cells, OPM1 cells were treated with andrographolide $(1,5$ or $10 \mu \mathrm{M})$ for 24,48 or $72 \mathrm{~h}$ and subjected to the MTT assay. As shown in Fig. 2, andrographolide significantly inhibited the proliferation of MM cells in vitro in a dose- and time-dependent manner (Fig. 2).

Andrographolide induces apoptosis of MM cells. To detect whether andrographolide induced apoptosis of MM cells, OPM1 cells were treated with andrographolide $(1,5$ or $10 \mu \mathrm{M})$ for $24 \mathrm{~h}$ and subjected to Annexin V/PI double staining followed by flow-cytometric evaluation. As shown in Fig. 3, andrographolide significantly induced apoptosis of MM cells in vitro in a dose-dependent manner.

Andrographolide induces caspase-9/3 activation of MM cells. To evaluate whether andrographolide induced caspase-9/3 activation in MM cells, OPM1 cells were treated with andrographolide $(1,5$ or $10 \mu \mathrm{M})$ for $24 \mathrm{~h}$ and subjected to a colorimetric ELISA assay. As shown in Fig. 4A and B, andrographolide effectively increased caspase-9/3 activation in $\mathrm{MM}$ cells in vitro in a dose-dependent manner (Fig. 4A and $\mathrm{B}$ )

Andrographolide inhibits TLR4 protein expression in MM cells. To further investigate the potential regulatory mechanisms of the effects exerted by andrographolide, the TLR4 protein expression of MM cells was determined using western blots analysis. As shown in Fig. 5A and B, andrographolide effectively reduced the levels of TLR4 protein expression in OPM1 cells in a dose-dependent manner (Fig. 5A and B).

Andrographolide inhibits $N F-\kappa B$ protein expression in $M M$ cells. To further elucidate the potential regulatory mechanism of andrographolide on the growth of $\mathrm{MM}$ cells, $\mathrm{NF}-\kappa \mathrm{B}$ protein expression in MM cells was detected using western blot analysis. As shown in Fig. 6A and B, andrographolide effectively reduced the level of $\mathrm{NF}-\kappa \mathrm{B}$ protein expression in OPM1 cells in a dose-dependent manner (Fig. 6A and B).

TLR4 siRNA enhances andrographolide-mediated inhibition of cell proliferation and induction of apoptosis of MM cells. To further confirm whether the TLR4/NF- $\kappa$ B signaling pathway was the functional target of andrographolide, TLR4 siRNA was transfected into MM cells. As shown in Fig. 7A and B, TLR4 siRNA inhibited the TLR4 protein expression in OPM1 cells. Of note, TLR4 siRNA efficiently enhanced the andrographolide-mediated inhibition of the cell proliferation and induction of apoptosis of MM cells (Fig. 7C and D). These results indicated that downregulation of TLR4 expression significantly enhanced the anti-cancer effects of andrographolide on MM cells.

TLR4 siRNA regulates $N F-\kappa B$ protein expression in $M M$ cells. To further confirm whether the TLR4/NF- $\kappa \mathrm{B}$ signaling pathway was the functional target of andrographolide, the $\mathrm{NF}-\kappa \mathrm{B}$ protein expression of MM cells was detected using western blot analysis. As shown in Fig. 8A and B, TLR4 siRNA significantly inhibited the expression of $N F-\kappa B$ protein in MM cells. The results indicated that downregulation of TLR4 expression significantly enhanced the inhibitory

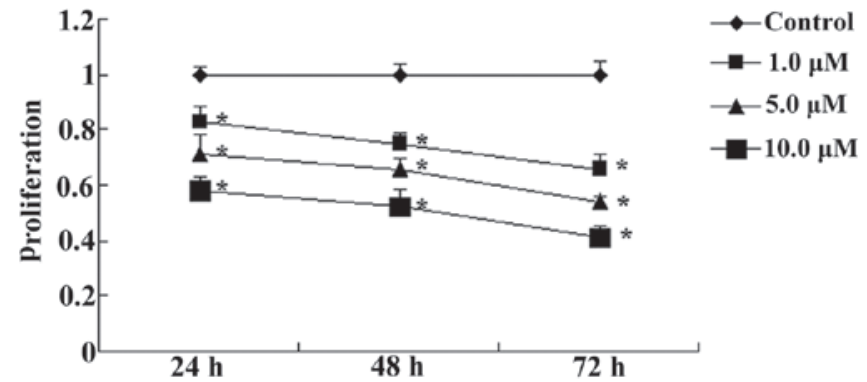

Figure 2. Andrographolide inhibits the proliferation of multiple myeloma cells Values are expressed as the mean \pm standard deviation. ${ }^{*} \mathrm{P}<0.01$ vs. control

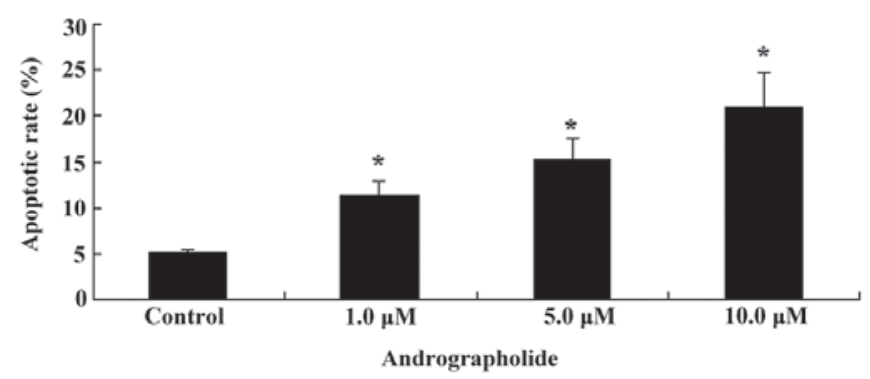

Figure 3. Andrographolide induces apoptosis of multiple myeloma cells. Values are expressed as the mean \pm standard deviation. ${ }^{*} \mathrm{P}<0.01$ vs. control.

effects of andrographolide on $\mathrm{NF}-\kappa \mathrm{B}$ expression in the MM cells.

$N F-\kappa B$ siRNA enhances andrographolide-mediated reduction of cell proliferation and induction of apoptosis of MM cells. To further assess whether the TLR4/NF- $\kappa \mathrm{B}$ signaling pathway is a functional target of andrographolide, $N F-\kappa B$ siRNA was transfected into MM cells. As shown in Fig. 9A and B, NF- $\kappa$ B siRNA inhibited the protein expression of $N F-\kappa B$ in $M M$ cells. Of note, $N F-\kappa B$ siRNA markedly enhanced andrographolide-mediated inhibition of cell proliferation and induction of apoptosis of MM cells (Fig. 9C and D). These results indicated that downregulation of $\mathrm{NF}-\kappa \mathrm{B}$ expression significantly enhanced the anti-cancer effects of andrographolide on MM cells.

\section{Discussion}

MM is a neoplasm of the blood, which is common in the elderly; its major cause is the proliferation of malignant plasma cells in the blood. With the increasing mean age of the Chinese population, the incidence of MM has been increasing (14). Autologous hematopoietic stem-cell transplantation can improve the number of normal blood plasma cells in cancer patients to prolong the survival time to a certain extent; however, as the age of patients with MM is generally high, stem-cell transplantation is not suitable for most of the patients (15). In the present study, andrographolide restrained the proliferation of MM cells in a dose- and time-dependent manner. Furthermore, andrographolide induced cellular apoptosis and caspase-9/3 activation in MM cells in a dose-dependent manner. Yang et al (16) reported that andrographolide induced apoptosis in glioma cells through the extracellular signal-regulated 

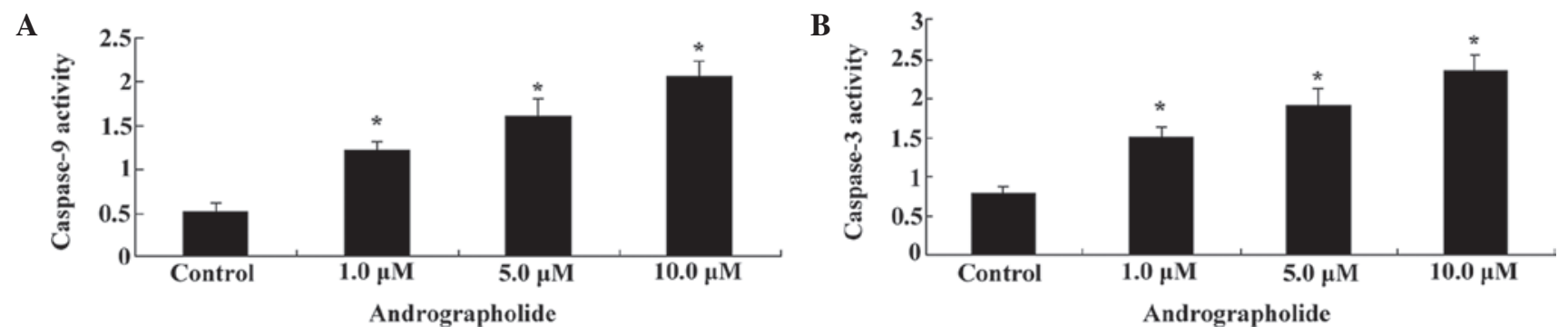

Figure 4. Andrographolide activates caspase-9/3 in multiple myeloma cells. (A) Caspase-9 and (B) caspase-3 expression levels. Values are expressed as the mean \pm standard deviation. ${ }^{*} \mathrm{P}<0.01$ vs. control.

A

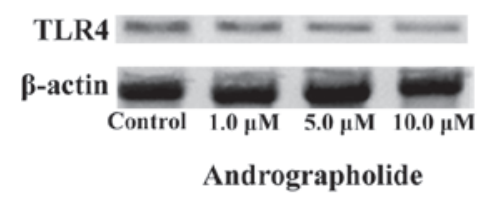

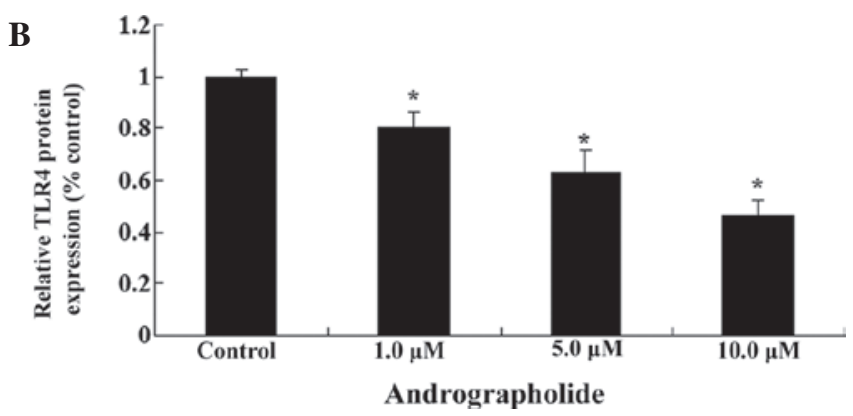

Figure 5. Andrographolide inhibits TLR4 protein expression in multiple myeloma cells. (A) Representative western blot showing TLR4 protein expression. (B) TLR4 expression was quantified by densitometric analysis with normalization to $\beta$-actin. Values are expressed as the mean \pm standard deviation. ${ }^{*} \mathrm{P}<0.01$ vs. control. TLR, Toll-like receptor.

A

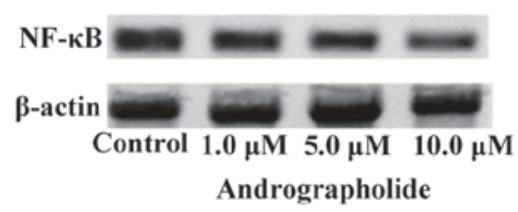

B

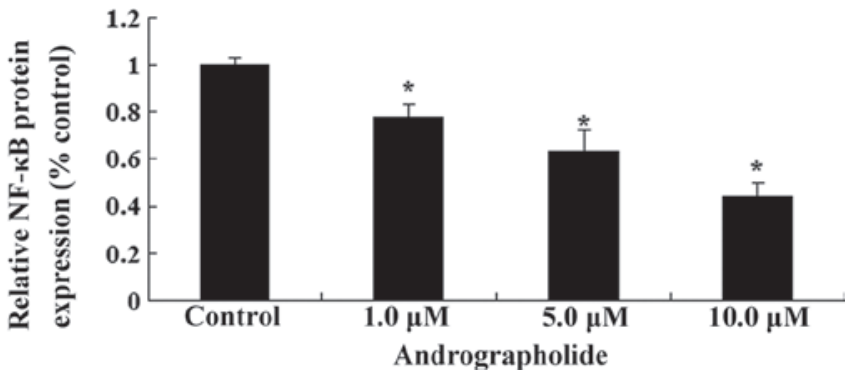

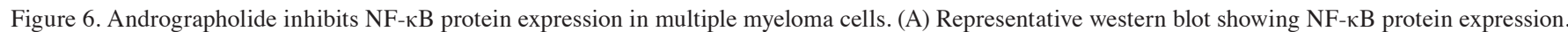
(B) NF- $\mathrm{kB}$ expression was quantified by densitometric analysis with normalization to $\beta$-actin. Values are expressed as the mean \pm standard deviation. ${ }^{*} \mathrm{P}<0.01$ vs. control. NF- $\kappa B$, nuclear factor $\kappa B$.

kinase/p53/caspase 7/poly(adenosine triphosphatase ribose) polymerase signaling pathway. Furthermore, andrographolide was shown to inhibit tumor angiogenesis through downregulation of vascular endothelial growth factor (VEGF)A/VEGF receptor 2 /mitogen-activated protein kinase pathway (17).

In humans, 11 types of TLRs have been identified, among which TLR4 was the first TLR found in mammals (3). Lipopolysaccharide (LPS) is the exogenous ligand of TLR4 and an in vivo study has shown that LPS stimulates the growth and metastasis of tumor cells (18). TLR4 is expressed in a variety of murine tumor-cell lines, and LPS-activated TLR4 signaling is conducive to tumor cells escaping from the microenvironment of immune surveillance; in addition, following siRNA-mediated TLR4 silencing, the inhibition of tumor cell growth was enhanced, which thus prolonged the survival time of mouse models with tumors (19). TLR4 is expressed on the surface of human ovarian cancer epithelial cells and can induce proliferation as well as enhance the production of cell cytokines following activation by LPS; therefore, it can be speculated that tumor cells regulate the tumor microenvironment via TLR4 and influence the activity of immune cells (20-22). The exogenous ligand of TLR4, LPS, promotes the proliferation of MM cells. Studies have revealed that andrographolide inhibits the growth of melanoma (23) and insulinoma (24) through inhibition of the TLR4/NF- $\mathrm{KB}$ signaling pathway. The present study demonstrated that andrographolide suppressed the protein expression levels of TLR4 in a dose-dependent manner. Furthermore, TLR4 siRNA enhanced andrographolide-mediated inhibition of cell proliferation and induction of apoptosis, while restraining the protein expression of NF- $\mathrm{\kappa B}$ in MM cells.

$\mathrm{NF}-\mathrm{\kappa B}$ is a protein which can specifically combine with $\kappa \mathrm{B}$ sites in a variety of gene promoters or enhancers to promote transcription (25). It regulates the expression of numerous genes, including cytokines, adhesion molecules, chemokines, immune factors, oxidative stress-associated 


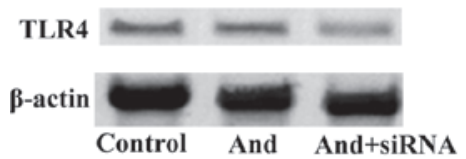

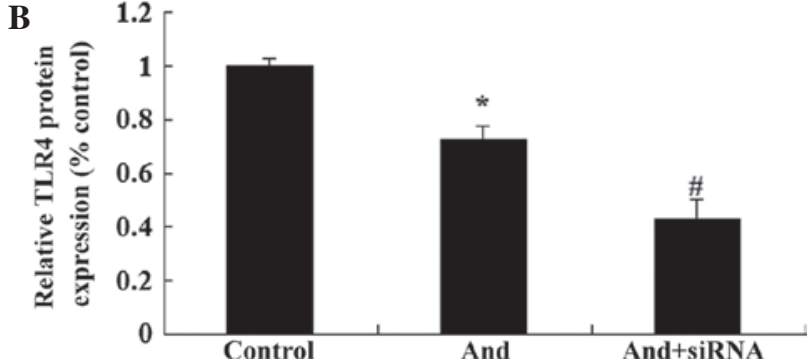

D
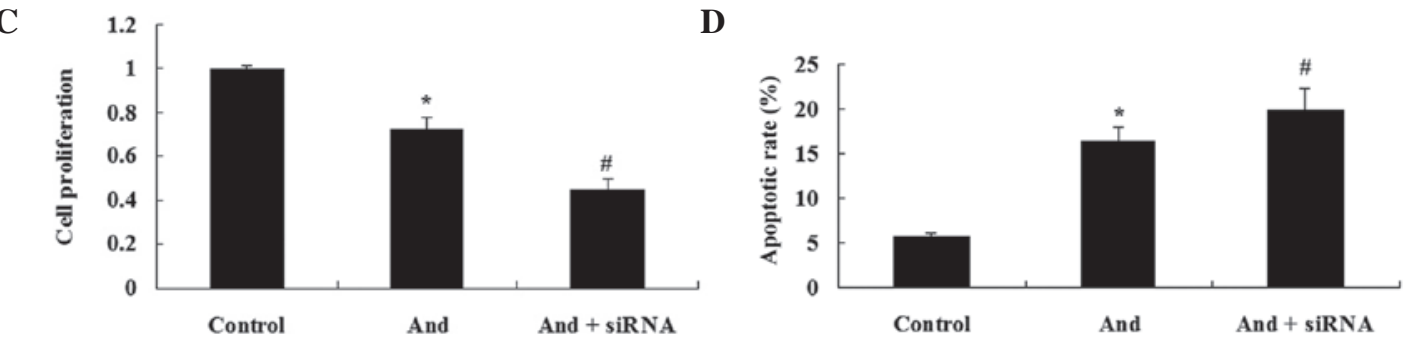

Figure 7. TLR4 siRNA regulates andrographolide-induced the cell proliferation and apoptosis of MM cells. (A) Representative western blot and (B) quantified expression levels obtained by densitometric analysis with normalization to $\beta$-actin confirming that TLR4 siRNA regulates TLR4 protein expression. (C) The reduction of cell proliferation and (D) the enhancement of apoptosis of MM cells by andrographolide were enhanced by TLR4 siRNA. Values are expressed as the mean \pm standard deviation. ${ }^{*} \mathrm{P}<0.01$ compared with control group, ${ }^{*} \mathrm{P}<0.01$ compared with $5.0 \mu \mathrm{M}$ andrographolide-treated group. MM, multiple myeloma; And, andrographolide; TLR, Toll-like receptor; siRNA small interfering RNA.
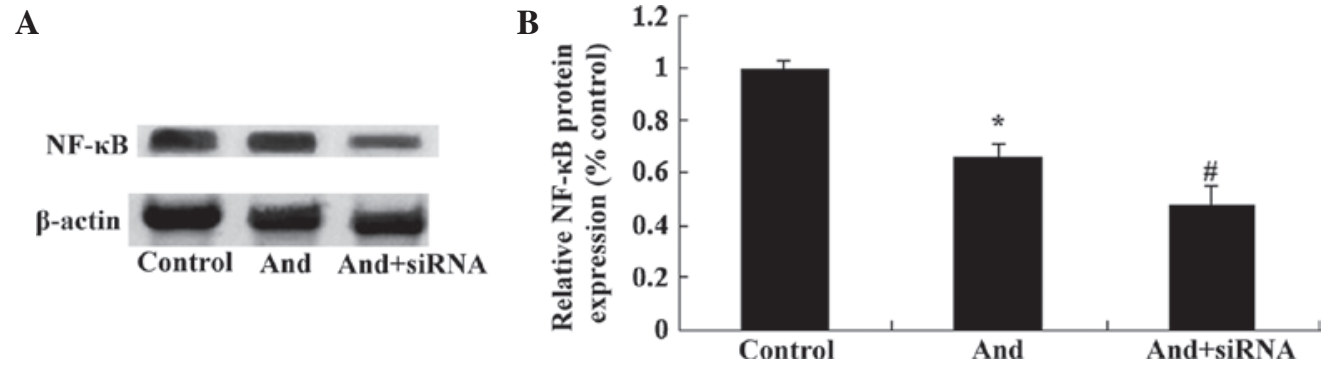

Figure 8. TLR4 siRNA regulates NF- $\mathrm{kB}$ protein expression of MM cells. (A) Representative western blots and (B) quantified expression levels obtained by densitometric analysis with normalization to $\beta$-actin confirming that TLR4 siRNA regulates NF- $\kappa \mathrm{B}$ protein expression in MM cells. Values are expressed as the mean \pm standard deviation. ${ }^{*} \mathrm{P}<0.01$ compared with control group, ${ }^{,} \mathrm{P}<0.01$ compared with $5.0 \mu \mathrm{M}$ andrographolide-treated group. MM, multiple myeloma; And, andrographolide; TLR, Toll-like receptor; siRNA small interfering RNA; NF-кB, nuclear factor kappa B.

A
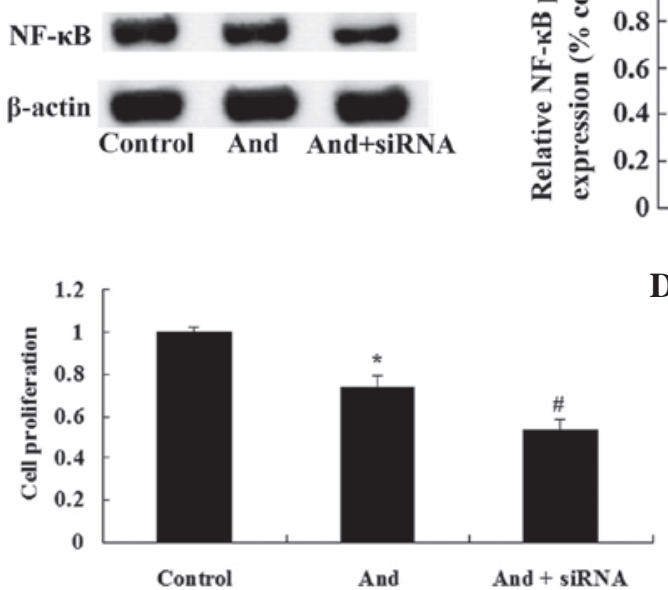

B

D
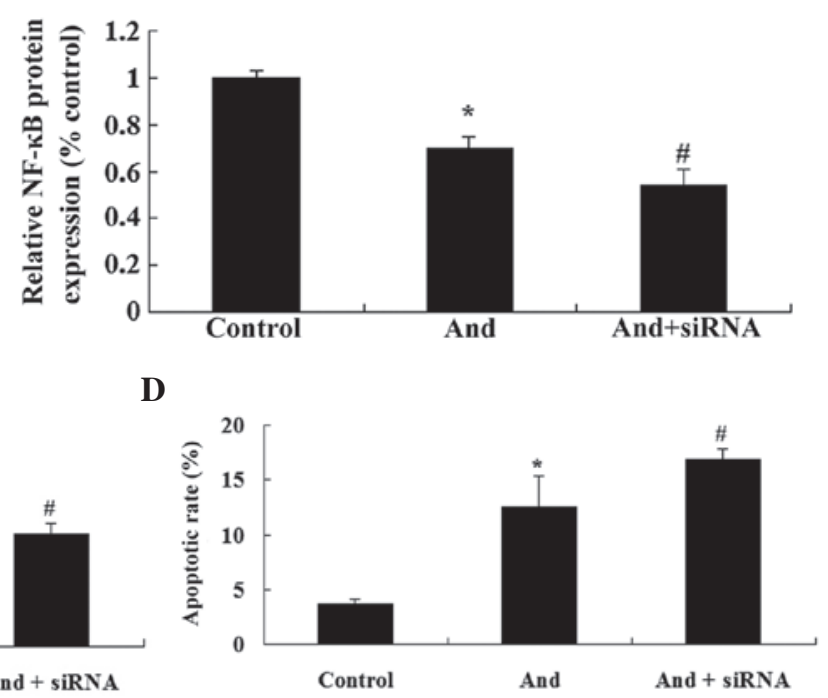

Figure 9. NF- $\mathrm{\kappa B}$ siRNA regulates andrographolide-mediated reduction of cell proliferation and enhancement if apoptosis of MM cells. (A) Representative western blots and (B) quantified expression levels obtained by densitometric analysis with normalization to $\beta$-actin confirming that NF- $\mathrm{KB}$ siRNA regulates NF- $\mathrm{kB}$ protein expression. (C) The reduction of cell proliferation and (D) the enhancement of apoptosis of MM cells by andrographolide were enhanced by NF- $\mathrm{kB}$ siRNA. Values are expressed as the mean \pm standard deviation. ${ }^{*} \mathrm{P}<0.01$ compared with control group, ${ }^{*} \mathrm{P}<0.01$ compared with $5.0 \mu \mathrm{M}$ andrographolide-treated group. MM, multiple myeloma; And, andrographolide; siRNA small interfering RNA; NF- $\mathrm{BB}$, nuclear factor kappa B. 
enzymes and transcription factors, and therefore has a variety of biological functions, including the participation in inflammatory immune responses, the regulation of cell apoptosis, self-transcription, cell cycle regulation, tumorigenesis and drug resistance $(26,27)$. As a transcription factor with a designated DNA-binding sequence, $\mathrm{NF}-\kappa \mathrm{B}$ has an important role in solid tumor-cell proliferation and transformation as well as tumor development; furthermore, it is closely associated with neoplasms of the blood system (28). Luo et al (13) showed that andrographolide inhibited the activation of $N F-\kappa B$ as well as matrix metalloproteinase-9 activity in H3255 lung cancer cells. Furthermore, Wang et al (29) demonstrated that andrographolide inhibited the proliferation of oral squamous cell carcinogenesis through inhibition of $N F-\kappa B$ inactivation. In the present study, andrographolide decreased the protein expression $N F-\kappa B$ in $M M$ cells. Of note, $N F-\kappa B$ siRNA significantly enhanced andrographolide-mediated inhibition of cell proliferation and induction of apoptosis of MM cells.

In conclusion, to the best of our knowledge, the present study was the first to show that andrographolide suppressed the proliferation and promoted apoptosis and caspase-9/3 activation in MM cells. The underlying mechanism may involve the suppression of the TLR4/NF- $\mathrm{B}$ signaling pathway. It is thus suggested that andrographolide may a promising candidate anti-cancer drug for the clinical treatment of MM through the TLR4/NF- $\kappa \mathrm{B}$ signaling pathway.

\section{References}

1. Auner HW, Szydlo R, Hoek J, Goldschmidt H, Stoppa AM, Morgan GJ, Moreau P, Attal M, Marit G, Russell N, et al: Trends in autologous hematopoietic cell transplantation for multiple myeloma in Europe: increased use and improved outcomes in elderly patients in recent years. Bone Marrow Transplant 50: 209-215, 2015.

2. Peng J, Chen Y, Lin J, Zhuang Q, Xu W, Hong Z and Sferra TJ: Patrinia scabiosaefolia extract suppresses proliferation and promotes apoptosis by inhibiting the STAT3 pathway in human multiple myeloma cells. Mol Med Rep 4: 313-318, 2011.

3. Li H, Xu H and Sun B: Lipopolysaccharide regulates MMP-9 expression through TLR4/NF- $\kappa \mathrm{B}$ signaling in human arterial smooth muscle cells. Mol Med Rep 6: 774-778, 2012.

4. Wang AC, Ma YB, Wu FX, Ma ZF, Liu NF, Gao R, Gao YS and Sheng XG: TLR4 induces tumor growth and inhibits paclitaxel activity in MyD88-positive human ovarian carcinoma in vitro. Oncol Lett 7: 871-877, 2014.

5. Murray MY, Zaitseva L, Auger MJ, Craig JI, MacEwan DJ, Rushworth SA and Bowles KM: Ibrutinib inhibits BTK-driven $\mathrm{NF}-\kappa \mathrm{B}$ p65 activity to overcome bortezomib-resistance in multiple myeloma. Cell Cycle 14: 2367-2375, 2015.

6. Dou A, Wang Z, Zhao J, Liu J and Zheng C: Identification of therapeutic target genes with DNA microarray in multiple myeloma cell line treated by IKK $\beta / N F-\kappa B$ inhibitor. Acta Cir Bras 29: 696-702, 2014.

7. Serrano FG, Tapia-Rojas C, Carvajal FJ, Hancke J, Cerpa W and Inestrosa NC: Andrographolide reduces cognitive impairment in young and mature A $\beta$ PPswe/PS-1 mice. Mol Neurodegener 9: 61, 2014.

8. Chua LS: Review on liver inflammation and antiinflammatory activity of Andrographis paniculata for hepatoprotection. Phytother Res 28: 1589-1598, 2014.

9. Yang D, Zhang W, Song L and Guo F: Andrographolide protects against cigarette smoke-induced lung inflammation through activation of heme oxygenase-1. J Biochem Mol Toxicol 27: 259-265, 2013.

10. Zeng X, Liu X, Bian J, Pei G, Dai H, Polyak SW, Song F, Ma L, Wang Y and Zhang L: Synergistic effect of 14-alpha-lipoyl andrographolide and various antibiotics on the formation of biofilms and production of exopolysaccharide and pyocyanin by Pseudomonas aeruginosa. Antimicrob Agents Chemother 55: 3015-3017, 2011
11. Lee JC, Tseng CK, Young KC, Sun HY, Wang SW, Chen WC, Lin CK and Wu YH: Andrographolide exerts anti-hepatitis $\mathrm{C}$ virus activity by up-regulating haeme oxygenase-1 via the p38 MAPK/Nrf2 pathway in human hepatoma cells. Br J Pharmacol 171: 237-252, 2014.

12. Lee C, Oh JI, Park J, Choi JH, Bae EK, Lee HJ, Jung WJ, Lee DS, Ahn KS and Yoon SS: TNF $\alpha$ mediated IL-6 secretion is regulated by JAK/STAT pathway but not by MEK phosphorylation and AKT phosphorylation in U266 multiple myeloma cells. Biomed Res Int 2013: 580135, 2013.

13. Luo W, Liu Y, Zhang J, Luo X, Lin C and Guo J: Andrographolide inhibits the activation of NF- $\mathrm{KB}$ and MMP-9 activity in $\mathrm{H} 3255$ lung cancer cells. Exp Ther Med 6: 743-746, 2013.

14. Yang M, Huang J, Ma QL, Xu GX and Jin J: Antitumor activity of CDA-, a urinary preparation, on human multiple myeloma cell lines via the mitochondrial pathway. Mol Med Rep 9: 1025-1031, 2014.

15. Corso A, Mangiacavalli S, Barbarano L, Montalbetti L, Mazzone A, Fava S, Varettoni M, Zappasodi P, Morra E and Lazzarino M: Low efficacy of thalidomide in improving response after induction in multiple myeloma patients who are candidates for high-dose therapy. Leuk Res 32: 1085-1090, 2008.

16. Yang SH, Wang SM, Syu JP, Chen Y, Wang SD, Peng YS, Kuo MF and Kung HN: Andrographolide induces apoptosis of C6 glioma cells via the ERK-p53-caspase 7-PARP pathway. Biomed Res Int 2014: 312847, 2014.

17. Shen K, Ji L, Lu B, Xu C, Gong C, Morahan G and Wang Z: Andrographolide inhibits tumor angiogenesis via blocking VEGFA/VEGFR2-MAPKs signaling cascade. Chem Biol Interact 218: 99-106, 2014.

18. Wang Y, He H, Li D, Zhu W, Duan K, Le Y, Liao Y and Ou Y: The role of the TLR4 signaling pathway in cognitive deficits following surgery in aged rats. Mol Med Rep 7: 1137-1142, 2013.

19. Bao H, Lu P, Li Y, Wang L, Li H, He D, Yang Y, Zhao Y, Yang L, Wang M, et al: Triggering of toll-like receptor-4 in human multiple myeloma cells promotes proliferation and alters cell responses to immune and chemotherapy drug attack. Cancer Biol Ther 11: 58-67, 2011.

20. Wang AC, Su QB, Wu FX, Zhang XL and Liu PS: Role of TLR4 for paclitaxel chemotherapy in human epithelial ovarian cancer cells. Eur J Clin Invest 39: 157-164, 2009.

21. Huang JM, Zhang GN, Shi Y, Zha X, Zhu Y, Wang MM, Lin Q, Wang W, Lu HY, Ma SQ, Cheng J and Deng BF: Atractylenolide-I sensitizes human ovarian cancer cells to paclitaxel by blocking activation of TLR4/MyD88-dependent pathway. Sci Rep 4: 3840, 2014.

22. Klink M,Nowak M,Kielbik M,BednarskaK,BlusE,Szpakowski M, Szyllo K and Sulowska Z: The interaction of HspA1A with TLR2 and TLR4 in the response of neutrophils induced by ovarian cancer cells in vitro. Cell Stress Chaperones 17: 661-674, 2012.

23. Zhang QQ, Zhou DL, Ding Y, Liu HY, Lei Y, Fang HY, Gu QL, He XD, Qi CL, Yang Y, et al: Andrographolide inhibits melanoma tumor growth by inactivating the TLR4/NF- $\kappa \mathrm{B}$ signaling pathway. Melanoma Res 24: 545-555, 2014.

24. Zhang QQ, Ding Y, Lei Y, Qi CL, He XD, Lan T, Li JC, Gong P, Yang X, Geng JG and Wang LJ: Andrographolide suppress tumor growth by inhibiting TLR4/NF- $\mathrm{BB}$ signaling activation in insulinoma. Int J Biol Sci 10: 404-414, 2014.

25. Suzuki E, Daniels TR, Helguera G, Penichet ML, Umezawa K and Bonavida B: Inhibition of NF-kappaB and Akt pathways by an antibody-avidin fusion protein sensitizes malignant B-cells to cisplatin-induced apoptosis. Int J Oncol 36: 1299-1307, 2010.

26. Calabrese C, Berman SH, Babish JG, Ma X, Shinto L, Dorr M, Wells K, Wenner CA and Standish LJ: A phase I trial of andrographolide in HIV positive patients and normal volunteers. Phytother Res 14: 333-338, 2000

27. Manni S, Brancalion A, Mandato E, Tubi LQ, Colpo A, Pizzi M, Cappellesso R, Zaffino F, Di Maggio SA, Cabrelle A, et al: Protein kinase CK2 inhibition down modulates the NF- $\kappa B$ and STAT3 survival pathways, enhances the cellular proteotoxic stress and synergistically boosts the cytotoxic effect of bortezomib on multiple myeloma and mantle cell lymphoma cells. PLoS One 8: e75280, 2013.

28. Siveen KS, Mustafa N, Li F, Kannaiyan R, Ahn KS, Kumar AP, Chng WJ and Sethi G: Thymoquinone overcomes chemoresistance and enhances the anticancer effects of bortezomib through abrogation of $\mathrm{NF}-\kappa \mathrm{B}$ regulated gene products in multiple myeloma xenograft mouse model. Oncotarget 5: 634-648, 2014.

29. Wang LJ, Zhou X, Wang W, Tang F, Qi CL, Yang X, Wu S, Lin YQ, Wang JT and Geng JG: Andrographolide inhibits oral squamous cell carcinogenesis through $\mathrm{NF}-\kappa \mathrm{B}$ inactivation. J Dent Res 90: 1246-1252, 2011. 